\title{
Thermal and chemical effects in shear and compaction bands
}

\author{
Jean Sulem, Ioannis Stefanou
}

Laboratoire Navier, CERMES, Ecole des Ponts ParisTech, 6, 8 Av. Blaise Pascal, 77455, Marne-la-Vallée, France

Localized deformation in the form of shear bands is one of the most common features of failure in geomaterials. Shear zones appear at all scales from tens of kilometres for large crustal faults, which accommodate tectonic deformation of the Earth's crust, to few microns for slip zones observed inside faults core. These very thin localized zones can sometimes accommodate tens of kilometres of slip. Although fault zones occupy only a small volume of the crust, they actually control most of its mechanical and fluid flow properties. They play a major role in controlling the migration of crustal fluids as for example the hydrocarbon migration, accumulation and leakage in sedimentary basins (Faulkner et al, 2010). The presence of faults and their dominant role in the control of regional fluid flow affects also other applications such as hydrocarbon recovery, deep waste storage repositories and geothermal systems. The study of earthquake mechanisms has shown that earthquakes appear to be the result of a frictional instability and occur by sudden slippage along a pre-existing fault or a plate interface (Scholz, 1998). In recent years, several drilling programs in active faults have highlighted the importance of the coupled physico-chemical processes involved in the nucleation of seismic slip (e.g. Niemeijer et al., 2012).

Another mode of failure commonly observed in geomaterials when subjected to compression is the formation of compaction bands in the direction perpendicular to the maximum principal stress, usually at stress states in the transitional regime between brittle faulting and cataclastic flow (Issen \& Rudnicki, 2000). As for faults, compaction bands significantly influence the stress field and fluid transport. Localized compaction in porous rocks dramatically reduces permeability across the compaction zone, providing highly anisotropic channelling of fluid flow in reservoir rocks (Holcomb et al., 2007). Change of permeability and compartmentalizing of fluid flow resulting from the presence deformation bands is thus of major importance in geo-energy systems.

Although strain localization in the form of shear band formation can occur with negative or positive rate of strain hardening, softening behaviour definitely favours shear banding (Rudnicki \& Rice, 1975, Vardoulakis \& Sulem, 1995). This softening behaviour may correspond to a mechanical degradation of the rock properties (microcracking, grain crushing and grain size reduction...), but various other physical processes can be responsible for it (Das et al., 2011). The effect of an infiltrated pore fluid which interacts with a rock mass can lead to a hardening or softening behaviour depending on the volumetric response of the rock (dilatant or contractant). The fast heating of a saturated geomaterial leads to pore-fluid pressurization due to the discrepancy between the thermal expansion of water and solid grains. Thermal pressurization is a softening mechanism as it results in a decrease of the effective mean stress and thus of the shear strength. Chemical reactions such as dissolution/ precipitation, mineral transformation at high temperature (dehydration of minerals, decomposition of carbonates, ...) affect the solid phase and the porosity of the rock and can induce a positive feedback in the progressive mechanical degradation. Thermal decomposition of minerals at large rise in temperature liberates a fluid product phase and enhances the generation of additional pore pressure excess (Sulem \& Famin, 2009, Brantut et al., 2011). It can form a mineral assemblage stronger (reaction hardening) or weaker (reaction weakening) than the original material (e.g. Castellanza \& Nova, 2004, Buscarnera, 2012). On the other hand, mechanical damage increases the reaction surface between a reactive fluid and the solid and enhances dissolution and further material weakening (Hu \& Hueckel, 2007a,b, Stefanou \& Sulem, 2014). 
As emphasized by Rice et al. (2014) the width of the deforming zone is a key parameter, as narrow deforming zones concentrate the frictional heating, which leads to large temperature rises and thus to more rapid weakening. The width of the deforming zone is determined by the various physical processes involved in the above weakening mechanisms but it also controls the multi-physics couplings which occur during dynamic slip. Obviously, this parameter plays also a major role in the energy budget of the system as it controls the feedback of the dissipative terms in the energy balance equation. Moreover, the width of the deforming zone may evolve in time as stronger softening favours a decrease of the shear band thickness, whereas heat and fluid diffusion tend to broaden the localized zone (Platt et al., 2014).

It is well known that strain localization analyses performed for rate-independent materials within the frame of classical continuum theories lead to infinitesimally narrow localized zones. This reflects the ill-posedness of the underlying mathematical problem and can be traced to the absence of a material length in the constitutive equations. Viscous regularization by considering strain rate hardening is commonly considered to overcome this problem. Another approach is to resort to continuum models with microstructure to describe, on a more physical basis, the localization phenomena. These generalized continua usually contain additional kinematical degrees of freedom (e.g. Cosserat continuum) and/or higher deformation gradients (higher grade continua). They introduce material internal lengths and also characteristic time scales. The localized zone thickness can be deduced from a linear stability analysis of the governing equations deduced from mass, energy and momentum balance. It depends on the constitutive, the hydraulic and the thermochemical properties of the deforming material. The internal length and the micro-inertia introduced from generalized continua permit to describe localization phenomena in zones of finite thickness and lead to a finite evolution rate of a deformation band like in strain rate dependent constitutive laws (Sulem et al. 2011; Veveakis et al. 2013).

If one considers the coupled effect of chemical softening, which results to further damage of the solid skeleton and to acceleration of the dissolution rate, due to the increase of the interface area between the reactive pore fluid and the solid phase, this positive feedback process may trigger instabilities in the form of compaction bands. In order to account for the heterogeneity of the dissolution process due to the heterogeneity of the microstructure of the material (e.g. different grain sizes and mineral constituents are present in the REV), non-local effects can be introduced in the chemical reaction rate as well as in the mechanical response. This approach naturally introduces an internal length that controls the thickness of these compaction bands (Stefanou \& Sulem, 2014).

The aforementioned examples aim to show how thermal and chemical effects dictate the stability, the energy budget and the evolution of several phenomena related to localized failure. Thermo-chemomechanical couplings are nowadays more and more identified as factors that play a central role in the mechanical behaviour and the evolution of localized deformation zones. Important challenges and questions are still open, which span from the qualitative understanding of the main phenomena to their quantitative description and observation. The current lecture focuses on presenting the general framework of localized failure in earthquake rupture, on the thermo-chemo-mechanical phenomena that take place at several time and length scales, on the existing theoretical interpretations and models and on some challenges for future investigations.

\section{References}

Brantut, N., Sulem, J., \& Schubnel, A. (2011). Effect of dehydration reactions on earthquake nucleation: Stable sliding, slow transients, and unstable slip. Journal of Geophysical Research: Solid Earth, 116, 1-16.

Buscarnera, G. (2012). A conceptual model for the chemo-mechanical degradation of granular geomaterials. Géotechnique Letters, 2 149-154.

Castellanza, R., \& Nova, R. (2004). Oedometric Tests on Artificially Weathered Carbonatic Soft Rocks. Journal of Geotechnical and Geoenvironmental Engineering, 130(7), 728-739. 
Das, A., Nguyen, G. D. \& Einav, I. (2011). "Compaction bands due to grain crushing in porous rocks: A theoretical approach based on breakage mechanics,” Journal of Geophysical Research, 2011, 116, no. B8, pp. 1-14.

Faulkner, D. R., Jackson, C. a. L., Lunn, R. J., Schlische, R. W., Shipton, Z. K., Wibberley, C. A. J., \& Withjack, M. O. (2010). A review of recent developments concerning the structure, mechanics and fluid flow properties of fault zones. Journal of Structural Geology, 32(11), 1557-1575.

Holcomb, D., Rudnicki, J. W., Issen, K. a., \& Sternlof, K. (2007). Compaction localization in the Earth and the laboratory: state of the research and research directions. Acta Geotechnica, 2(1), 1-15.

Hu, L.-B., \& Hueckel, T. (2007). Creep of saturated materials as a chemically enhanced ratedependent damage process, 31, 1537-1565.

Hu, L.-B., \& Hueckel, T. (2007). Coupled chemo-mechanics of intergranular contact: Toward a threescale model. Computers and Geotechnics, 34(4), 306-327.

Issen, K. a., \& Rudnicki, J. W. (2000). Conditions for compaction bands in porous rock. Journal of Geophysical Research, 105(B9), 21529.

Niemeijer, A., Di Toro, G., Griffith, A. W., Bistacchi, A., Smith, S. a F., \& Nielsen, S. (2012). Inferring earthquake physics and chemistry using an integrated field and laboratory approach. Journal of Structural Geology, 39, 2-36.

Platt, J. D., Rudnicki, J. W., \& Rice, J. R. (2014). Stability and localization of rapid shear in fluidsaturated fault gouge: 2. Localized zone width and strength evolution. Journal of Geophysical Research: Solid Earth, 119(5), 4334-4359.

Rice, J. R. (2006). Heating and weakening of faults during earthquake slip. Journal of Geophysical Research, 111(B5), B05311.

Rice, J. R., Rudnicki, J. W., \& Platt, J. D. (2014). Stability and localization of rapid shear in fluidsaturated fault gouge: 1. Linearized stability analysis. Journal of Geophysical Research: Solid Earth, 119(5), 4311-4333. Rudnicki, J. W., \& Rice, J. R. (1975). Conditions for the localization of deformation in pressure-sensitive dilatant materials. Journal of the Mechanics and Physics of Solids, 23, 371-394.

Scholz, C. H. (1998). “Earthquakes and friction laws,” Nature, 1998, 391, pp. 37-42.

Stefanou, I. \& Sulem, J. (2014). "Chemically induced compaction bands: Triggering conditions and band thickness,” Journal of Geophysical Research: Solid Earth, 2014, 119, no. 2, pp. 880-899.

Sulem, J., \& Famin, V. (2009). Thermal decomposition of carbonates in fault zones: Slip-weakening and temperature-limiting effects. Journal of Geophysical Research, 114(B3), B03309.

Sulem, J., Stefanou, I., \& Veveakis, E. (2011). Stability analysis of undrained adiabatic shearing of a rock layer with Cosserat microstructure. Granular Matter, 13(3), 261-268. doi:10.1007/s10035-0100244-1

Veveakis, E., Stefanou, I., \& Sulem, J. (2013). Failure in shear bands for granular materials: thermohydro-chemo-mechanical effects. Géotechnique Letters, 3(April-June), 31-36. 